\title{
DNA copy number aberrations associated with aneuploidy and chromosomal instability in breast cancers
}

\author{
SHIGETO KAWAUCHI ${ }^{1}$, TOMOKO FURUYA ${ }^{1}$, KENZO IKEMOTO $^{1}$, MOTONAO NAKAO $^{1}$, \\ SHIGERU YAMAMOTO $^{2}$, MASAAKI OKA ${ }^{2}$ and KOHSUKE SASAKI ${ }^{1}$ \\ Departments of ${ }^{1}$ Pathology and ${ }^{2}$ Surgery, Yamaguchi University Graduate School of Medicine, \\ Ube, Yamaguchi 755-8505, Japan
}

Received May 8, 2010; Accepted June 21, 2010

DOI: $10.3892 /$ or_00000933

\begin{abstract}
Biological characteristics of a tumor are primarily affected by its genomic alterations. It is thus important to ascertain whether there are genomic changes linked with DNA ploidy and/or chromosomal instability (CIN). In the present study, using fresh-frozen samples of 46 invasive breast cancers, laser scanning cytometry, array-based comparative genomic hybridization, and chromosome fluorescence in situ hybridization were performed to assess DNA ploidy, DNA copy number aberrations (DCNAs), and CIN status. Both ploidy and CIN status were examined in 36 tumors, resulting in 23 aneuploid/CIN ${ }^{+}$tumors, 1 aneuploid/ $\mathrm{CIN}^{-}, 2 \mathrm{diploid} / \mathrm{CIN}^{+}$, and $10 \mathrm{diploid} / \mathrm{CIN}^{-}$tumors. Comparison of the aCGH data with the DNA ploidy and CIN status identified cytogenetically 11 characteristic breast cancers with distinctive DCNAs. The 11 tumors were classified into two types; one type is diploid/CIN- phenotype containing 4 DCNAs, and the other aneuploid/ $\mathrm{CIN}^{+}$phenotype containing 7 DCNAs. In $30(65.2 \%)$ of the 36 breast cancers, the status of DNA ploidy and CIN depended on the type of DCNAs. Furthermore, the DNA ploidy phenotype depended on the dominant type of DCNAs even in tumors with a mixture of multiple DCNAs of one type and a single DCNA of the other type. Tumors with multiple DCNAs of both types represented aneuploidy and over three quarters of breast cancers carry at least one type of the DCNAs. These results suggested that, in breast cancers, the status of DNA ploidy and CIN was likely to determine at the beginning of carcinogenesis.
\end{abstract}

\section{Introduction}

Cancer cells possess extensive genomic alterations, ranging from intragenic mutations, to gross gains and losses of chromo-

Correspondence to: Dr Shigeto Kawauchi, Department of Pathology, Yamaguchi University Graduate School of Medicine, Ube, Yamaguchi 755-8505, Japan

E-mail: shig@yamaguchi-u.ac.jp

Key words: breast cancer, DNA copy number aberration, arraybased comparative genomic hybridization, chromosomal instability, tumor classification somal material $(1,2)$. Aneuploidy, an alteration in the number of chromosomes or nuclear DNA content, is a common trait of tumor cells, and it drives tumor progression (3). With tumor progression, aneuploidy successively confers a more aggressive character to tumor cells (4). Thus, aneuploidy has been used as a prognostic marker of various kinds of cancers including breast cancer (5-7).

Genomic instability is also recognized as inherent characteristics of cancer cells. The underlying mechanisms of genomic instability are different among tumors. Genomic instability is roughly divided into microsatellite instability (MIN) and chromosomal instability (CIN) (8). CIN affords tumor cells the ability to make continual changes in their chromosome numbers and structures with deletions and amplifications of chromosomal regions, and as a result, it provides cancer cells with further structural and numerical abnormalities of chromosomes (8-11).

A close relationship has been suggested between aneuploidy and CIN $(8,9)$. Aneuploidy and CIN usually coexist in tumor cells (8) and aneuploidy is considered to be a surrogate marker of CIN (9). However, aneuploidy is conceptually different from CIN (9). In normal cells, both ploidy and genomic stability are strictly maintained, and are rigorously controlled by complex mechanisms with hundreds of genes related to mitosis and DNA repair. The defects of the mechanisms potentially cause abnormal mitosis and genetic alterations, leading to aneuploidy and/or genomic instability (12). There are multiple mechanisms to confer aneuploidy and CIN on a tumor cell (13). Tumor phenotype including aneuploidy and genomic instability is thus primarily affected by their genomic changes.

The identification of genomic changes associated with aneuploidy and CIN is crucial to elucidate the evolutional mechanisms of aneuploidy and CIN. However, information on the relationship between genomic alterations and the status of ploidy and genomic instability is scarce. First, a comprehensive analysis of genomic changes in tumors is necessary, because tumors with aneuploidy or CIN represent genomic abnormalities at many chromosomal regions. Genomic alterations such as DNA copy number aberrations (DCNAs) linked with the status of DNA ploidy and CIN have not been identified. In particular, it is necessary to clarify whether there are genomic aberrations that differ between diploid and aneuploid tumors, and between $\mathrm{CIN}^{-}$and $\mathrm{CIN}^{+}$tumors, 
and whether there are any genomic alterations that are shared by aneuploidy and $\mathrm{CIN}^{+}$.

In the present study, we identified DCNAs associated with both aneuploidy and CIN in breast cancers, and the DCNA pattern allowed classification of breast cancers into two types, aneuploid/CIN ${ }^{+}$and diploid/CIN- tumors. The purpose of the study was to assess the extent of the genomic aberrations observed in tumors with aneuploidy and CIN, and to gain insight into the evolutional mechanisms of aneuploidy and CIN in tumors.

\section{Materials and methods}

Tumor tissue specimens. The present study analyzed 46 primary invasive breast cancers. All tumors were considered to be sporadic. The average age of the patients was 57.6 years, ranging from 31 to 75 years. None of the patients had received either chemotherapy or radiation prior to surgery. The Institutional Review Board for human tissue use at Yamaguchi University Graduate School of Medicine approved the study protocol, and informed consent for this study was obtained from all the patients. A representative part of the surgically removed tumor tissue specimens was used for touch-smear preparations before fixation. The tumor tissue specimens were fixed in $10 \%$ buffered formalin for one day and then were subjected to a histological examination including an immunohistochemical study. The touch-smear preparations were subjected to fluorescence in situ hybridization (FISH) and laser scanning cytometry (LSC).

FISH and assessment of CIN. CIN was examined by fluorescence in situ hybridization (FISH) using 4 pericentromeric probes (chromosomes 7, 11, 17, and 18 for D7Z1, D11Z1, D17Z1, and D18Z1, respectively; Abbott Laboratories. Abbott Park, IL) on the touch-smear preparations in 36 of 46 tumors as described previously (14-16), and the presence or absence of CIN was determined according to the degree of variations in the number of FISH spots between nuclei (14-16). The tumors were considered to be CIN positive $(\mathrm{CIN}+)$ when the fraction of cells with modal chromosome number was $<75 \%$ for four chromosomes.

DNA ploidy determination. Measurement of nuclear DNA content by LSC was performed in 46 breast cancer specimens. Samples were made and DNA ploidy was determined by the procedures described previously $(14,15)$. Briefly, the touch-smear preparations fixed in $70 \%$ ethanol were dipped in a propidium iodide solution $(25 \mu \mathrm{g} / \mathrm{ml}$ in PBS) containing 0.1\% RNase (Sigma-Aldrich Co., St. Louis, MO). The DNA content was measured by a laser scanning cytometer (LSC 101; Olympus, Japan). In each touch smear sample $>5,000$ cells were examined. A DNA histogram was generated, and DNA ploidy was determined. DNA ploidy was expressed by the DNA index (DI). A case with $1.0 \leq \mathrm{DI}<1.2$ was classified as a diploid, and others were classified as aneuploid tumors $(14,15)$.

Array-based comparative gemonic hybridization. Arraybased comparative genomic (aCGH) was performed for 46 breast cancers. A tissue microdissection technique was used to reduce the contamination of samples by normal tissue components for aCGH analyses, as previously described (17). The normal cell contamination of the samples was reduced as much as possible, and it was usually $<10 \%$. High-molecular-weight DNA was extracted from each microdissected tumor specimen with a DNA extraction kit (SepaGene, Sankojyunyaku Co., Tokyo, Japan) according to the manufacturer's instructions. The BAC DNA array used in this study consists of 4030 human bacterial artificial chromosome (BAC) clones, including 356 cancer-related genes, which are spaced $\sim 0.83 \mathrm{Mb}$ across the whole genome (Macrogen, Inc., Seoul, Korea). BAC chip information is available on the following websites (http://www.macrogen. co.kr/eng/biochip/karyo_summary.jsp).

The experiments were performed as previously described (18-20). Briefly, tumor DNA (500 ng) and gender-matched reference DNA (Promega, Madison, WI) were labeled with Cy5- and Cy3-dCTP (Perkin-Elmer Life Science, Inc., Waltham, MA), respectively, with a random primer labeling kit (BioPrine ${ }^{\circledR}$ DNA Labelling System, Invitrogen, Carlsbad, CA). Labeled DNA was mixed with Cot-1 DNA (50 mg, Gibco-BRL, Gaithersburg, MD) and the probe mixture that was denatured at $75^{\circ} \mathrm{C}$ was applied to the array. After hybridization, slides were scanned on a GenePix 4000A scanner (Axon Instruments, Union City, CA) and the 16-bit TIFF images captured using GenePix Pro 5.0 software. Fluorescence images were analyzed with MAC Viewer ${ }^{\mathrm{TM}}$ software (Macrogen Inc.) optimized for analysis of the array. Fluorescence spots were defined with the automatic grid feature and adjusted manually. Thereafter the ratio of the red/green channel of each clone was calculated log base 2 transformed $\left(\log _{2}\right.$ ratios). The clones with $\log _{2}$ ratios exceeding \pm 0.25 were considered gain and loss of the copy number. A $\log _{2}$ ratio $>1.0$ was defined as amplifications in this study (18-20).

Statistical analysis. A clone-by-clone comparison of the copy number was made between the cell lines and tumor tissues. The differences in the prevalence of common gains and losses between two groups were determined with the $\chi^{2}$ test. A difference was considered to be significant when the p-value was $<0.05$.

\section{Results}

DNA ploidy and CIN. Fifteen (32.6\%) of 46 tumors were classified as diploid and $31(67.4 \%)$ were classified as aneuploid tumors. Twenty-five $(69.4 \%)$ of 36 tumors examined by FISH 25 were $\mathrm{CIN}^{+}$, and $11(30.6 \%)$ were CIN-. Therefore, aneuploid/CIN ${ }^{+}$, aneuploid/CIN ${ }^{-}$, diploid/CIN ${ }^{+}$, and diploid/CIN- tumors accounted for $23,1,2$, and 10 of the specimens, respectively.

$a C G H$. DCNAs were examined in 46 tumors. Gains of $1 \mathrm{q}$ and $8 \mathrm{q}$, and losses of $8 \mathrm{p}, 16 \mathrm{q}$, and $17 \mathrm{p}$ were detected in $>50 \%$ of 46 tumors (Fig. 1). BAC clones mapped to the broad area 1q23-q44 represented the highest frequency of copy number gains, and in particular, the gain of 1q32.1 (clone 2893) was detected in $78.3 \%$ of 46 tumors (Table IA). The gain of 8q23.3 was detected in $30(66.7 \%)$ tumors. The 


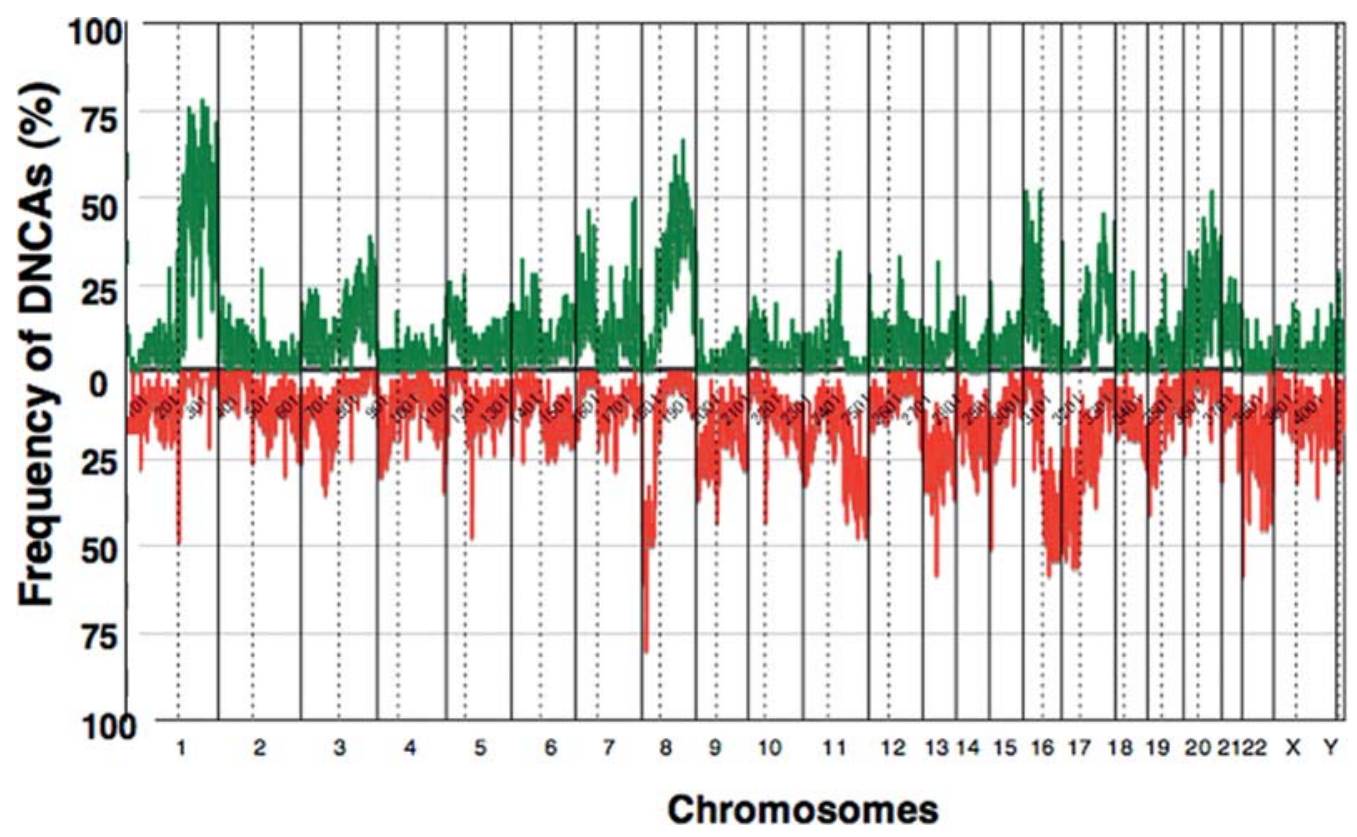

Figure 1. The frequency of DCNAs for each spot of the array spotted with 4030 BAC clones. Gains of 1q and 8q, and losses of $8 \mathrm{p}$, 16q, and $17 \mathrm{p}$ are detected in $>50 \%$ of tumors. BAC clones mapped to the broad area 1q23-q44 display the high frequency of copy number gains, and the gain of $1 \mathrm{q} 32.1$ is detected as frequent as in $78.3 \%$ of 46 tumors. The gain of $8 \mathrm{q} 23.3$ is detected in $30(66.7 \%)$ tumors. A copy number loss of $8 \mathrm{p} 23.1$ is detected in 37 (80.4\%) tumors and a copy number loss of 8 p23.3 is detected in $28(60.9 \%)$ tumors.

copy number loss of 8p23.1 (clone 4589) and 8p23.3 (clones 5579 and 923) was detected in $37(80.4 \%)$ and $28(60.9 \%)$ tumors, respectively (Table IB).

$a C G H$ vs. DNA ploidy. DCNAs were observed to be linked with the DNA ploidy status as shown in Table II. Gains of 1q31.1 (clone 347), 1q21.1 (1319), and 1q24.2 (1071) were more frequent in diploid than in aneuploid tumors $(11 / 13$ vs. $6 / 31,10 / 15$ vs. $3 / 31$, and $11 / 15$ vs. $7 / 31$, respectively), whereas gains of $19 q 13.2$ (2307), 20q13.33 (4092), and $12 q 15$ (2084), and 7p14.1 (2549) were preferentially detected in aneuploid tumors (0/15 vs. 13/31 for diploid vs. aneuploid tumors, $1 / 15$ vs. $13 / 31,0 / 14$ vs. $10 / 30$, and $2 / 15$ vs. $16 / 31$, respectively). A loss of 16q23.1 (clone 4264) was more frequent in diploid than in aneuploid tumors (11/15 vs. 4/31), whereas a loss of $17 \mathrm{p} 13.1$ (75) was preferentially detected in aneuploid tumors (1/15 vs. 22/31 for diploid vs. aneuploid tumors).

aCGH vs. CIN. Both aCGH and FISH were applied to 36 tumors. Gains of 1q21.1 (clone 1319), 1q24.2 (1071), 16 p13.11 (2311), and 1q31.1 (347) were more frequent in $\mathrm{CIN}^{-}$than in $\mathrm{CIN}^{+}$tumors (7/11 vs. $3 / 25,8 / 11$ vs. $6 / 25,3 / 11$ vs. $0 / 25$, and $7 / 9$ vs. $7 / 25$, respectively), whereas gains of 19q13.2 (2307), and 7p14.1 (2549) were frequently detected more in $\mathrm{CIN}^{-}$than in $\mathrm{CIN}^{+}$tumors (0/11 vs. $11 / 25$, and $1 / 11$ vs. $14 / 25$, respectively). A loss of 16q23.1 (4264) was more frequent in $\mathrm{CIN}^{-}$than in $\mathrm{CIN}^{+}$tumors (0/11 vs. 13/25), whereas 14q24.3 (1546), 22q13.2 (2842), and 1p36.22 (296) were preferentially detected in $\mathrm{CIN}^{+}(0 / 11$ vs. $13 / 25$ for $\mathrm{CIN}^{-}$vs. $\mathrm{CIN}^{+}$tumors, $0 / 11$ vs. $12 / 25$, and $0 / 11$ vs. 11/25, respectively, Table III).
DCNAs vs. DNA ploidy and CIN. DCNAs and the ploidy and CIN were examined in 36 tumors. The comparison of DCNAs with both the ploidy and CIN status identified 11 DCNAs that were associated with both the DNA ploidy and CIN status. At least one of these DCNAs was detected in 34 tumors, though none of these DCNAs were detected in one diploid/CIN- tumor and one aneuploid/CIN ${ }^{+}$tumor (Table IV).

Four DCNAs composed of gains of 1q31.1, 1q21.1, and $1 \mathrm{q} 24.2$ and loss of $16 \mathrm{q} 23.1$ were more frequent in diploid/CIN ${ }^{-}$than in aneuploid/CIN ${ }^{+}$tumors (7/10 vs. 5/23, $7 / 10$ vs. $1 / 23,7 / 10$ vs. $4 / 23$, and $7 / 10$ vs. $3 / 23$, respectively, Table IV). One to 4 of these DCNAs were observed in the 10 diploid/CIN- tumors and in both of the 2 diploid/CIN ${ }^{+}$ tumors (Table IV). Accordingly, these genomic alterations are conveniently referred to as 'diploid/CIN- DCNAs'. The 'diploid/ CIN' DCNAs' were also observed in some of the aneuploid/CIN ${ }^{+}$tumors (Table IV).

In contrast, 7 DCNAs composed of gains 19q13.2, 7p14.1, 20q13.33, and losses 17p13.1, 1p36.22, 22q13.2, and $4 q 13.3$ were detected exclusively in aneuploid tumors. One to 6 of these DCNAs were found in all 23 aneuploid/CIN ${ }^{+}$ and 1 aneuploid/CIN- tumor. Accordingly, these alterations were conveniently referred to as 'aneuploid/CIN ${ }^{+}$DCNAs'. One of the 'aneuploid/CIN ${ }^{+}$DCNAs' was detected in only 3 diploid/CIN- tumors (Table IV). There were aneuploid/ $\mathrm{CIN}^{+}$tumors that carried both diploid/CIN- and aneuploid/ $\mathrm{CIN}^{+}$DCNAs, whereas diploid/CIN- tumors barely carried aneuploid/CIN ${ }^{+}$DCNAs.

Ploidy v.s. 'diploid/CIN-DCNAs' and 'aneuploid/CIN ${ }^{+}$ DCNAs'. A comparison of the ploidy status and DCNAs was 
Table I. Clones representing frequent copy number gain and loss in 46 breast cancers.

A, Clones representing frequent copy number gain.

\begin{tabular}{llll}
\hline Clone ID & Chromosome region & & Genes \\
\hline 2893 & $1 \mathrm{q} 32.1$ & NFASC, & 78.3 \\
2316 & $1 \mathrm{q} 23.3$ & FCGR3A, FCGR3B, FCGR2B, FCRLM1, FCRLM2 & 76.1 \\
4752 & $1 \mathrm{q} 32.1$ & IL24, FAIM3, PIGR, FCAMR & 76.1 \\
1365 & $1 \mathrm{q} 42.12$ & CNIH3 & 76.1 \\
1038 & $1 \mathrm{q} 25.2$ & ASTN & 73.9 \\
4527 & $1 \mathrm{q} 41$ & DUSP10 & 73.9 \\
1078 & $1 \mathrm{q} 32.3$ & SLC30A1, NEK2 & 71.7 \\
5826 & $1 \mathrm{q} 44$ & OR2T4, OR2T6, OR2T1 & 71.7 \\
1344 & $1 \mathrm{q} 25.3$ & C1orf19 & 69.6 \\
2715 & $1 \mathrm{q} 32.1$ & BTG2, FMOD & 69.6 \\
4483 & $1 \mathrm{q} 32.2$ & PLXNA2 & 69.6 \\
4819 & $1 \mathrm{q} 41$ & KCNK2 & 69.6 \\
2271 & $1 \mathrm{q} 32.1$ & CNTN2, TMEM81, RBBP5, RIPK5 & 68.9 \\
2520 & $1 \mathrm{q} 32.1$ & MDM4, LRRN5, & 67.4 \\
2197 & $1 \mathrm{q} 44$ & SH3BP5L, ZNF672, ZNF692 \\
1394 & $8 \mathrm{q} 23.3$ & N.I. & 67.4 \\
1412 & $1 \mathrm{q} 23.2$ & PIGM, KCNJ10 & 66.7 \\
4858 & $1 \mathrm{q} 42.13$ & OBSCN & 65.2 \\
4710 & $1 \mathrm{q} 42.2$ & SLC35F3 & 65.2 \\
5559 & $1 \mathrm{q} 44$ & OR2T12, OR2M7, OR5BF1, OR2T4, OR2T6, OR2T1 & 65.2 \\
\hline
\end{tabular}

$\mathrm{B}$, Clones representing frequent copy number loss.

\begin{tabular}{|c|c|c|c|}
\hline Clone ID & Chromosome region & Genes & Frequency $(\%)$ \\
\hline 4589 & $8 \mathrm{p} 23.1$ & FAM90A6P, FAM90A7 & 80.4 \\
\hline 5579 & $8 \mathrm{p} 23.3$ & ERICH1, C8orf68 & 60.9 \\
\hline 923 & $8 \mathrm{p} 23.3$ & N.I. & 58.7 \\
\hline 1601 & $16 q 21$ & TK2, CKLF, CMTM1 & 58.7 \\
\hline 4916 & $13 \mathrm{q} 21.1$ & N. I. & 58.6 \\
\hline 613 & $17 \mathrm{p} 13.1$ & $\begin{array}{l}\text { ASGR1, DLG4, ACADVL, DVL2, PHF23, } \\
\text { GABARAP, DULLARD, C17orf81, CLDN7, } \\
\text { SLC2A4, YBX2, EIF5A, GPS2 }\end{array}$ & 56.5 \\
\hline 2226 & $17 \mathrm{p} 12$ & ELAC2 & 56.5 \\
\hline 5831 & $8 \mathrm{p} 23.3$ & ERICH1 & 54.3 \\
\hline 2868 & $16 q 22.1$ & C16orf70, TRADD, FBXL8, HSF4, NOL3, E2F4, ELMO3 & 54.3 \\
\hline 965 & $16 \mathrm{q} 22.3$ & ATBF1 & 54.3 \\
\hline 1070 & $16 \mathrm{q} 23.1$ & WWOX & 54.3 \\
\hline 4128 & $16 \mathrm{q} 24.2$ & FBXO31 & 54.3 \\
\hline 1146 & $16 \mathrm{q} 24.3$ & ANKRD11 & 54.3 \\
\hline 5652 & $17 \mathrm{p} 13.3$ & ABR, MRPL14P1 & 54.3 \\
\hline 1142 & $16 \mathrm{q} 21$ & N. I. & 53.3 \\
\hline 1536 & $17 \mathrm{p} 13.1$ & MYH3, SCO1, C17orf48 & 53.3 \\
\hline 1536 & $17 \mathrm{p} 13.1$ & MYH3, SCO1, C17orf48 & 53.3 \\
\hline 5643 & $8 \mathrm{p} 23.3$ & C8orf68 & 52.2 \\
\hline 2281 & $16 q 24.1$ & WFDC1 & 52.2 \\
\hline 5816 & $16 \mathrm{q} 24.3$ & AFG3L1, DBNDD1, GAS8, C16orf3, PRDM7 & 52.2 \\
\hline 1597 & $17 \mathrm{p} 11.2$ & FBXW10, FAM18B & 52.2 \\
\hline
\end{tabular}

N.I., not identified. 
Table II. Clones with significant difference in frequency of copy number loss between diploid and aneuploid breast cancers.

\begin{tabular}{|c|c|c|c|c|c|c|c|c|c|}
\hline \multirow{3}{*}{$\begin{array}{l}\text { Clone } \\
\text { ID }\end{array}$} & \multicolumn{3}{|c|}{ Copy number gain } & \multirow[b]{3}{*}{ P-value } & \multirow{3}{*}{$\begin{array}{l}\text { Clone } \\
\text { ID }\end{array}$} & \multicolumn{3}{|c|}{ Copy number loss } & \multirow[b]{3}{*}{ P-value } \\
\hline & \multirow{2}{*}{$\begin{array}{l}\text { Chromosome } \\
\text { region }\end{array}$} & \multicolumn{2}{|c|}{ Frequency } & & & \multirow{2}{*}{$\begin{array}{l}\text { Chromosome } \\
\text { region }\end{array}$} & \multicolumn{2}{|c|}{ Frequency } & \\
\hline & & Diploid & Aneuploid & & & & Diploid & Aneuploid & \\
\hline 347 & $1 \mathrm{q} 25$ & $11 / 13$ & $6 / 31$ & 0.0000498699 & 4264 & $16 \mathrm{q} 23.1$ & $11 / 15$ & $4 / 31$ & 0.0000415712 \\
\hline 1319 & $1 \mathrm{q} 21.1$ & $10 / 15$ & $3 / 31$ & 0.0000571916 & 75 & $17 \mathrm{p} 13.1$ & $1 / 15$ & $22 / 31$ & 0.0000433612 \\
\hline 1071 & $1 \mathrm{q} 24.2$ & $11 / 15$ & $7 / 31$ & 0.0009451900 & 1597 & $17 \mathrm{p} 11.2$ & $2 / 15$ & $21 / 31$ & 0.0005406710 \\
\hline 2307 & $19 \mathrm{q} 13.2$ & $0 / 15$ & $13 / 31$ & 0.0030650620 & 430 & $17 \mathrm{p} 13.3$ & $1 / 15$ & $18 / 31$ & 0.0009037900 \\
\hline 4092 & $20 q 13.33$ & $1 / 15$ & $13 / 31$ & 0.0148092780 & 2934 & 17 p12 & $1 / 15$ & $18 / 31$ & 0.0009037900 \\
\hline 2084 & $12 q 15$ & $0 / 14$ & $10 / 30$ & 0.0139915010 & 1564 & $17 \mathrm{p} 11.2$ & $1 / 15$ & $18 / 31$ & 0.0009037900 \\
\hline \multirow[t]{7}{*}{2549} & $7 \mathrm{p} 14.1$ & $2 / 15$ & $16 / 31$ & 0.0126392840 & 2226 & $17 \mathrm{p} 12$ & $3 / 15$ & $22 / 31$ & 0.0011408200 \\
\hline & & & & & 5095 & $17 \mathrm{p} 13.3$ & $2 / 15$ & $20 / 31$ & 0.0011231510 \\
\hline & & & & & 2801 & $17 \mathrm{p} 13.1$ & $2 / 15$ & $20 / 31$ & 0.0011231510 \\
\hline & & & & & 4504 & $17 \mathrm{p} 12$ & $2 / 15$ & $20 / 31$ & 0.0011231510 \\
\hline & & & & & 296 & $1 \mathrm{p} 36.22$ & $0 / 15$ & $15 / 31$ & 0.0010315680 \\
\hline & & & & & 2308 & $16 q 13$ & $12 / 15$ & $9 / 31$ & 0.0011408200 \\
\hline & & & & & 4999 & $16 \mathrm{q} 21$ & $10 / 15$ & $6 / 31$ & 0.0015868770 \\
\hline
\end{tabular}

Table III. Clones with significant difference in frequency of copy number loss between CIN positive and negative breast cancers.

\begin{tabular}{|c|c|c|c|c|c|c|c|c|c|}
\hline \multirow{3}{*}{$\begin{array}{l}\text { Clone } \\
\text { ID }\end{array}$} & \multicolumn{3}{|c|}{ Copy number gain } & \multirow[b]{3}{*}{ P-value } & \multirow{3}{*}{$\begin{array}{l}\text { Clone } \\
\text { ID }\end{array}$} & \multicolumn{3}{|c|}{ Copy number loss } & \multirow[b]{3}{*}{ P-value } \\
\hline & \multirow{2}{*}{$\begin{array}{l}\text { Chromosome } \\
\text { region }\end{array}$} & \multicolumn{2}{|c|}{ Frequency } & & & \multirow{2}{*}{$\begin{array}{l}\text { Chromosome } \\
\text { region }\end{array}$} & \multicolumn{2}{|c|}{ Frequency } & \\
\hline & & $\mathrm{CIN}^{-}$ & $\mathrm{CIN}^{+}$ & & & & $\mathrm{CIN}^{-}$ & $\mathrm{CIN}^{+}$ & \\
\hline 1319 & $1 q 21.1$ & $7 / 11$ & $3 / 25$ & 0.001441054 & 1546 & $14 q 24.3$ & $0 / 11$ & $13 / 25$ & 0.002770077 \\
\hline 1071 & 1q24.2 & $8 / 11$ & $6 / 25$ & 0.005734709 & 2842 & $22 q 13.2$ & $0 / 11$ & $12 / 25$ & 0.004889128 \\
\hline 2311 & $16 \mathrm{p} 13.11$ & $3 / 11$ & $0 / 25$ & 0.006386024 & 296 & $1 \mathrm{p} 36.22$ & $0 / 11$ & $11 / 25$ & 0.008290603 \\
\hline 2307 & $19 q 13.2$ & $0 / 11$ & $11 / 25$ & 0.008290603 & 4561 & $7 q 21.11$ & $3 / 11$ & $0 / 25$ & 0.006386024 \\
\hline 2549 & $7 \mathrm{p} 14.1$ & $1 / 11$ & $14 / 25$ & 0.008544027 & 75 & $17 \mathrm{p} 13.1$ & $2 / 11$ & $16 / 25$ & 0.011318931 \\
\hline 347 & $1 q 25$ & $7 / 9$ & $7 / 25$ & 0.009271438 & 4264 & $16 \mathrm{q} 23.1$ & $7 / 11$ & $5 / 25$ & 0.010515247 \\
\hline 4079 & $3 \mathrm{p} 24.3$ & $0 / 11$ & $10 / 24$ & 0.011305418 & & & & & \\
\hline 2069 & $2 \mathrm{p} 25.3$ & $0 / 11$ & $10 / 25$ & 0.013577166 & & & & & \\
\hline
\end{tabular}

made in 46 tumors. Although 1 (tumor No. 15) of 15 diploids carried none of the 11 DCNAs, 14 of 15 DNA diploid tumors represented at least 1 of 'diploid/CIN- DCNAs' (Fig. 3). Of these 14 diploid tumors, 10 had only 'diploid/CIN- DCNAs', and the remaining 4 diploid tumors (Nos. 1, 4, 7, and 9) carried 'diploid/CIN' DCNAs' and 1 of 'aneuploid/CIN ${ }^{+}$ DCNAs' (Fig. 3).

One (No. 125) of 31 aneuploid tumors carried none of 11 DCNAs, though 20 had only 'aneuploid/CIN ${ }^{+}$DCNAs', and 5 aneuploid tumors had 'aneuploid/CIN ${ }^{+}$DCNAs' and 1 of 'diploid/CIN' DCNAs' (Fig. 2). Three aneuploid tumors (Nos.
104, 107, and 126) had 3 'aneuploid/CIN+ DCNAs' and 4 'diploid/CIN' DCNAs', 1 aneuploid tumor (No. 108) had 3 'aneuploid/CIN ${ }^{+}$DCNAs' and 4 'diploid/CIN' DCNAs', and 1 aneuploid tumor (No. 114) had 7 'aneuploid/CIN ${ }^{+}$DCNAs' and 2 'diploid/CIN' DCNAs' (Fig. 3). All these 5 tumors in which multiple diploid/CIN- DCNAs and multiple aneuploid/ $\mathrm{CIN}^{+}$DCNA coexisted showed an aneuploid phenotype (Fig. 2). These tumors made up $\sim 10 \%$ of aneuploid cancers. Multiple aneuploid/CIN ${ }^{+}$DCNAs conferred an aneuploid phenotype to breast cancers with or without diploid/CINDCNAs. 
Table IV. BAC clones of which DNA copy number aberrations are associated with ploidy/CIN status.

\begin{tabular}{|c|c|c|c|c|c|c|c|}
\hline $\begin{array}{l}\text { Clone ID } \\
\text { tumors }\end{array}$ & $\begin{array}{l}\text { Chromosome } \\
\text { region }\end{array}$ & Genes & $\mathrm{A} / \mathrm{CIN}^{+}$ & $\mathrm{A} / \mathrm{CIN}^{-}$ & $\mathrm{D} / \mathrm{CIN}^{+}$ & $\mathrm{D} / \mathrm{CIN}^{-}$ & $\begin{array}{l}\text { No. of } \\
\text { tumors }\end{array}$ \\
\hline \multicolumn{8}{|c|}{ Copy number gain } \\
\hline 347 & 1q31.1 & TPR, C1orf27, OCLM, PDC & 5 & 0 & 2 & 7 & 14 \\
\hline 1319 & $1 \mathrm{q} 21.1$ & GJA8 & 1 & 0 & 2 & 7 & 10 \\
\hline 1071 & 1q24.2 & PRRX1 & 4 & 0 & 2 & 8 & 14 \\
\hline 2307 & $19 \mathrm{q} 13.2$ & $\begin{array}{l}\text { IL28A, IL29, LRFN1, GMFG, } \\
\text { SAMD4B }\end{array}$ & 11 & 0 & 0 & 0 & 11 \\
\hline 2549 & $7 \mathrm{p} 14.1$ & $\begin{array}{l}\text { TRG@, TRGJP1, TRGV11, } \\
\text { TRGVB, TRGV10, TRGV9, } \\
\text { TRGVA, TRGV8, TRGV7, }\end{array}$ & & & & & \\
\hline & & TRGV6, TRGV5P, TRGV5, TRGV4 & 14 & 0 & 0 & 1 & 15 \\
\hline 4092 & $20 q 13.33$ & LAMA5, RPS21, CABLES2, C20orf151 & 12 & 0 & 0 & 1 & 13 \\
\hline \multicolumn{8}{|c|}{ Copy number loss } \\
\hline 4264 & $16 q 23.1$ & N.I. & 3 & 0 & 2 & 7 & 12 \\
\hline 75 & $17 \mathrm{p} 13.1$ & GLP2R, RCV1, GAS7 & 16 & 1 & 0 & 1 & 18 \\
\hline 296 & $1 \mathrm{p} 36.22$ & FRAP1, ANGPTL7, UBIAD1 & 11 & 0 & 0 & 0 & 11 \\
\hline 2842 & $22 \mathrm{q} 13.2$ & XRCC6, NHP2L1, HMG17L2 & 12 & 0 & 0 & 0 & 12 \\
\hline 2490 & $4 q 13.3$ & PF4, PPBP, CXCL5, CXCL3, PPBPL2 & 10 & 0 & 0 & 0 & 10 \\
\hline No. of tumors & & & 23 & 1 & 2 & 10 & 36 \\
\hline
\end{tabular}

N. I., not identified; A, aneuploidy; D, diploidy.

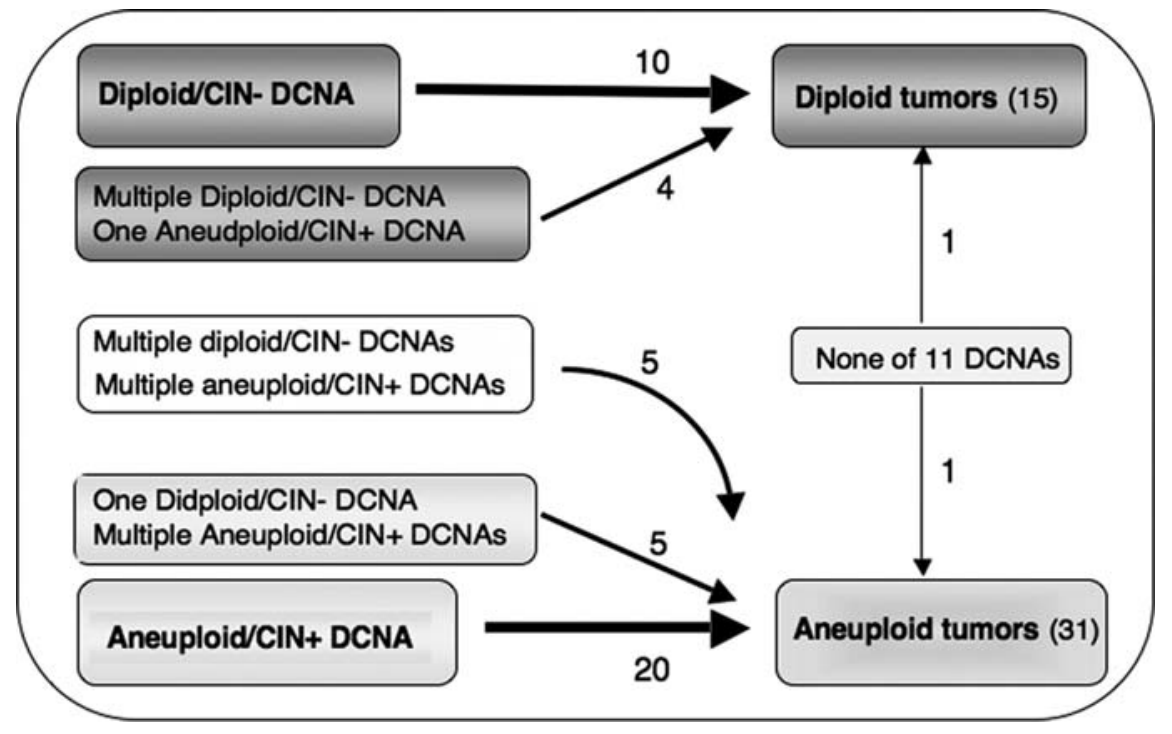

Figure 2. The diagram shows the relationship between DCNAs and ploidy in 46 breast cancers consisting of 15 diploid and 31 aneuploid tumors. In total, 11 DCNAs are identified to show differentiation between diploid and aneuploid cancers. Four DCNAs, gains of 1q31.1, 1q21.1, and 1q24.2 and loss of 16q23.1 are associated with a diploid phenotype, referred to as 'diploid/CIN- DCNAs'. Seven DCNAs, gains 19q13.2, 7p14.1, 20q13.33, and losses 17p13.1, 1p36.22, 22q13.2, and 4q13.3 are associated with an aneuploid phenotype, referred to as 'aneuploid/CIN ${ }^{+}$DCNAs'. Tumors are classified according to the types of DCNAs. Ten diploid cancers carry only diploid/CIN- DCNAs, whereas 20 aneuploid cancers carry only aneuploid/CIN ${ }^{+}$DCNAs. Tumors with diploid/CIN ${ }^{-}$ DCNAs and a single aneuploid/CIN ${ }^{+}$DCNAs show a diploid phenotype, and 4 tumors are included in this category. In turn, tumors with aneuploid/CIN ${ }^{+}$ DCNAs and a single diploid/CIN- DCNAs show an aneuploid phenotype, and 5 tumors are included in this category. All tumors with multiple DCNAs for both types show aneuploid phenotype, indicating that aneuploid phenotype is a dominant trait to diploid one, and 5 tumors are included in this category. This suggests that some aneuploid tumors occur through diploid ones. Diploid tumors do not occur through aneuploid tumors, because aneuploidy is a dominant phenotype. Neither type of DCNAs is detected in 1 of diploid tumors and 1 of aneuploid tumors. As a result, the diploid-aneuploid tumor ratio is $1: 2$ in breast cancers $(15: 31)$. The figures adjacent to arrows indicate the number of tumors according to each criterion. 

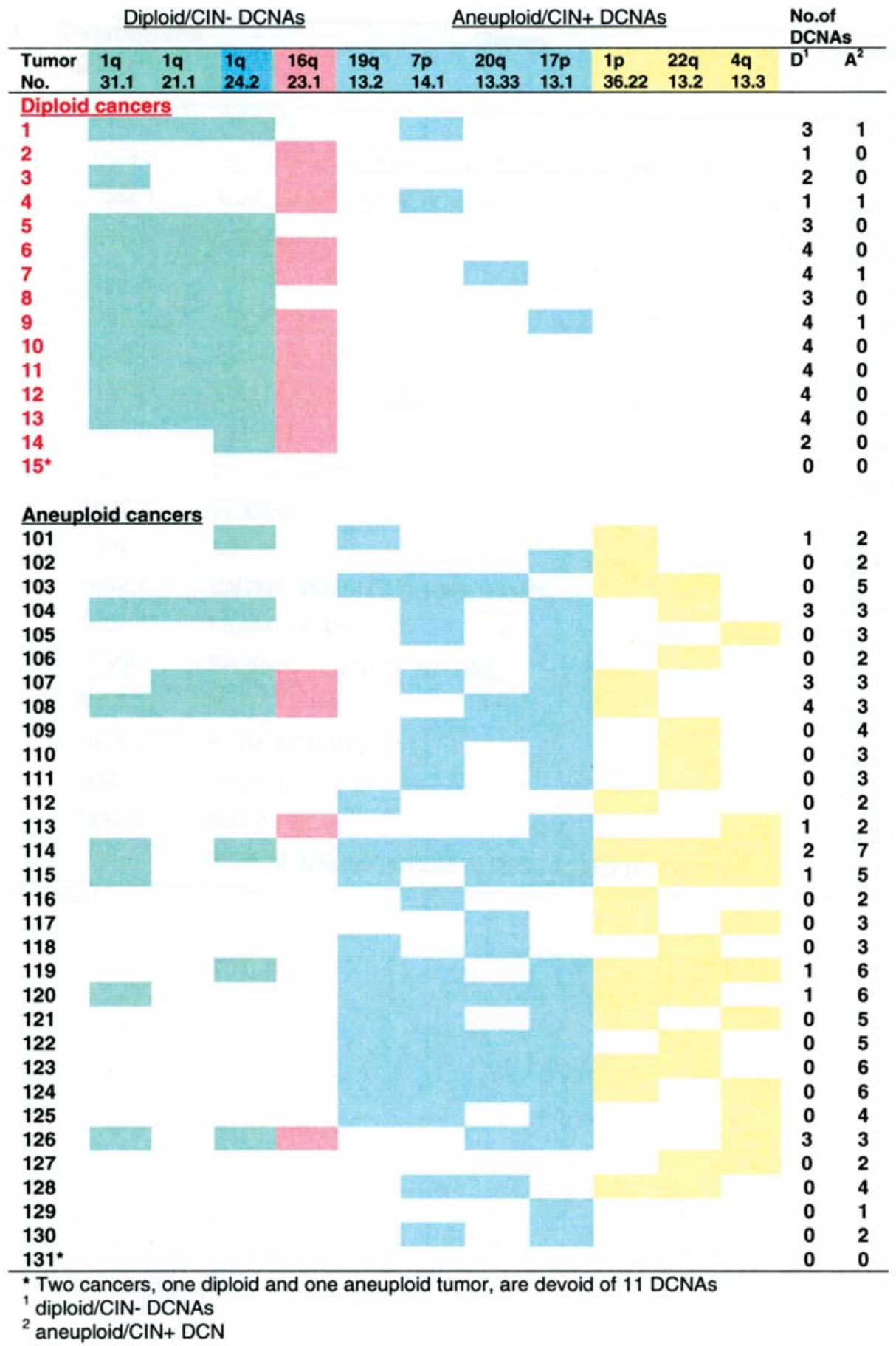

Figure 3. Four 'diploid/CIN- DCNAs' and 7 'aneuploid/CIN ${ }^{+}$DCNAs' in diploid and aneuploid breast cancers.

\section{Discussion}

DNA aneuploidy is frequently observed in malignant tumors, and it accounts for two thirds of breast cancers (21-23). In this study, the frequency of aneuploidy was $67.4 \%$, and this is consistent with the previous data. Although aneuploidy is not equated with CIN, aneuploidy is recognized as a surrogate marker of CIN $(8,9)$. The present study revealed that aneuploid tumors frequently overlapped with $\mathrm{CIN}^{+}$ ones and diploid tumors frequently overlapped with CIN ones. The biological characteristics of a tumor are primarily affected by genomic alterations of the tumor cells. These raise a question whether aneuploidy and $\mathrm{CIN}^{+}$share a common evolutionary mechanism.

The present aCGH study allowed for the identification of 11 DCNAs with a differing frequency between diploid and aneuploid cancers and between $\mathrm{CIN}^{-}$and $\mathrm{CIN}^{+}$cancers. Of these DCNAs, 4 were linked with a diploid/CIN- phenotype, whereas 7 were linked with an aneuploid/CIN ${ }^{+}$phenotype. Breast cancers were divided into two categories according to the combination of these two DCNA types; tumors with either one type of DCNAs or the other, and tumors with both types of DCNAs. In $30(65.2 \%)$ breast cancers with either one type of DCNAs or the other, the phenotypic status of 
ploidy and CIN depends on the relevant type of DCNAs. Tumors with only 'diploid/CIN- DCNAs' represented a diploid/CIN' phenotype, while tumors with only 'aneuploid/ $\mathrm{CIN}^{+}$DCNAs' represented an aneuploid/CIN ${ }^{+}$phenotype. In tumors with both types of DCNAs, however, the relationship between DCNA type and ploidy/CIN status may be somewhat complicated.

Tumors with multiple diploid/CIN- DCNAs and a single aneuploid/CIN ${ }^{+}$DCNA showed a diploid phenotype. In reverse cases, an aneuploid phenotype was always observed. Therefore, the ploidy phenotype depends on the dominant type of DCNAs in tumors with a mixture of a single DCNA of one type and multiple DCNAs of the other type. Interestingly, all tumors that carry multiple diploid/CIN- DCNAs and multiple aneuploid/CIN ${ }^{+}$DCNAs showed an aneuploid phenotype. These findings suggest that both DNA ploidy and genomic instability are determined at the beginning of carcinogenesis, and that 'aneuploid/CIN ${ }^{+}$DCNAs' are phenotypically dominant over the diploid/CIN- ones. This is consistent with the hypothesis that the aneuploid/ $\mathrm{CIN}^{+}$ phenotype is dominant over diploid/CIN- one $(8,9)$.

Aneuploid/ $\mathrm{CIN}^{+}$tumors were observed to carry both diploid/CIN ${ }^{-}$and aneuploid/CIN ${ }^{+}$DCNAs, whereas diploid/ $\mathrm{CIN}^{-}$tumors rarely carried aneuploid/CIN ${ }^{+}$DCNAs. Therefore, it is legitimate to consider that some aneuploid tumors (5 tumors in this series) occur through diploid ones during the course of tumor progression. However, diploid tumors do not occur through aneuploid ones, because aneuploidy is a dominant phenotype. In other words, around $10 \%$ of aneuploid tumors evolve through diploid tumors. Aneuploid/ $\mathrm{CIN}^{+}$tumors can therefore be divided into two types; $a b$ initio aneuploid/CIN ${ }^{+}$and secondary aneuploid/CIN ${ }^{+}$tumors. The developmental rate of a diploid tumor may thus be similar to that of aneuploid tumors, but the diploid-to-aneuploid transition in tumors makes the diploid/aneuploid tumor ratio around $1 / 2$ as shown in clinical samples.

Genomic instability is roughly divided into microsatellite instability (MIN) and CIN, which are considered to be the underlying mechanisms responsible for diploidy and aneuploidy, respectively $(8,9,24,25)$. However, there is little information about genomic alterations implicated in both ploidy and CIN status. Two types of DCNAs, 'diploid/CINDCNAs' and 'aneuploid/CIN+ DCNAs' were identified for differentiating diploid/CIN' and aneuploid/CIN ${ }^{+}$tumors . The identification of DCNAs linked with both ploidy and CIN status raises the hypothesis that there is an evolutional pathway shared by ploidy and CIN status. It is easy to explain why the association between aneuploidy and $\mathrm{CIN}^{+}$is a usual event in tumors, assuming that a common pathway generates both aneuploidy and $\mathrm{CIN}^{+}$. Aneuploidy and $\mathrm{CIN}^{+}$phenotypes are accompanied by checkpoint silencing $(26,27)$ and are generated by genetic aberrations targeting components of mitotic process (26-28). However, seven chromosomal regions with 'aneuploid/CIN ${ }^{+}$DCNAs', 1p36.22, 4q13.3, 7p14.1, 19q13.2, 20q13.33, 17p13.1, and 22q13.2, harbor no genes directly related to a checkpoint. Another mechanism is therefore required to explain the association between aneuploidy and $\mathrm{CIN}^{+}$.

Other mechanisms that have not yet been identified influence checkpoint activity (29), and there are genes encoding modulators of genomic and mitotic stability (30-32). If the chromosomal regions with these DCNAs harbor genes modulating whole suites of downstream genes at various points in pathways for the maintenance of genome and chromosome, it is not difficult to suppose that the DCNAs would affect genomic and mitotic stability. When at least 1 of 7 'aneuploid/CIN ${ }^{+}$DCNAs' is added to the basic genomic changes in a tumor cell, the cell ends up showing aneuploidy and $\mathrm{CIN}^{+}$phenotype. It is not yet possible to identify which genes on these chromosomal regions with DCNAs are involved in the status of ploidy and CIN, nor is it possible to explain how the genes are involved in the status of ploidy and CIN.

In conclusion, the present study revealed 11 DCNAs linked with the status of ploidy and CIN in breast cancers. Four DCNAs were linked with the diploid/CIN- phenotype, whereas 7 DCNAs were linked with the aneuploid/CIN ${ }^{+}$phenotype. These two types of DCNAs represented a mutually exclusive relationship between diploid and aneuploidy and between the $\mathrm{CIN}^{-}$and $\mathrm{CIN}^{+}$phenotypes. In particular, the identification of these 7 DCNAs may thus provide valuable new insights into the mechanism behind the development of aneuploidy/CIN ${ }^{+}$. The present $\mathrm{CGH}$ study may contribute to the discovery a novel pathway for the aneuploidy/CIN ${ }^{+}$ phenotype. Further research is required to elucidate this new mechanism for aneuploidization and CIN.

\section{Acknowledgements}

This study was supported in part by The Ministry of Education, Culture of Japan (21390106 and 21659088) and The New Energy and Industrial Technology Development Organization (NEDO) of Japan. We acknowledge Takae Okada for providing us with technical assistance.

\section{References}

1. Marx J: Debate surges over the origins of genomic defects in cancer. Science 297: 544-546, 2002.

2. Sadikovic B, Al-Romaih K, Squire JA and Zielenska M: Cause and consequences of genetic and epigenetic alterations in human cancer. Curr Genomics 9: 394-408, 2008.

3. Holland AJ and Cleveland DW: Boveri revisited: chromosomal instability, aneuploidy and tumorigenesis. Nat Rev Mol Cell Biol 10: 478-487, 2009.

4. Bayani J, Selvarajah S, Maire G, Vukovic B, Al-Romaih K, Zielenska $M$ and Squire JA: Genomic mechanisms and measurement of structural and numerical instability in cancer cells. Semin Cancer Biol 17: 5-18, 2007.

5. Haroske G, Baak JP, Danielsen H, et al: Fourth updated ESACP consensus report on diagnostic DNA image cytometry. Anal Cell Pathol 23: 89-95, 2001.

6. Millot $C$ and Dufer J: Clinical applications of image cytometry to human tumour analysis. Histol Histopathol 15: 1185-2000, 2000.

7. Suehiro Y, Okada T, Okada T, et al: Aneuploidy predicts outcome in patients with endometrial carcinoma and is related to lack of CDH13 hypermethylation. Clin Cancer Res 14: 3354-3361, 2008.

8. Lengauer C, Kinzler KW and Vogelstein B: Genetic instability in colorectal cancers. Nature 386: 623-627, 1997.

9. Lengauer C, Kinzler KW and Vogelstein B: Genetic instabilities in human cancers. Nature 396: 643-649, 1998.

10. Maser RS and DePinho RA: Take care of your chromosomes lest cancer take care of you. Cancer Cell 3: 4-6, 2003.

11. Rajagopalan H, Nowak MA, Vogelstein B and Lengauer C: The significance of unstable chromosomes in colorectal cancer. Nat Rev Cancer 3: 695-701, 2003. 
12. Kops GJ, Weaver BA and Cleveland DW: On the road to cancer: aneuploidy and the mitotic checkpoint. Nat Rev Cancer 5: 773-785, 2005 .

13. Shen C, Zhou Y, Zhan J, Reske SN and Buck AK: Chromosome instability and tumor lethality suppression in carcinogenesis. J Cell Biochem 105: 1327-1341, 2008.

14. Miyazaki M, Furuya T, Shiraki A, Sato T, Oga A and Sasaki K: The relationship of DNA ploidy to chromosomal instability in primary human colorectal cancers. Cancer Res 59: 5283-5285, 1999.

15. Furuya T, Uchiyama $\mathrm{T}$ and Murakami T: Relationship between chromosomal instability and intratumoral regional DNA ploidy heterogeneity in primary gastric cancers. Clin Cancer Res 6: 2815-2820, 2000

16. Yamamoto Y, Matsuyama H, Kawauchi S, et al: Biological characteristics in bladder cancer depend on the type of genetic instability. Clin Cancer Res 12: 2752-2758, 2006.

17. Hashimoto Y, Oga A, Okami K, Imate Y, Yamashita Y and Sasaki K: Relationship between cytogenetic aberrations by CGH coupled with tissue microdissection and DNA ploidy by laser scanning cytometry in head and neck squamous cell carcinoma. Cytometry 40: 161-166, 2000.

18. Furuya T, Uchiyama T, Adachi A, et al: The development of a mini-array for estimating the disease states of gastric adenocarcinoma by array CGH. BMC Cancer 8: 393, 2008.

19. Chochi Y, Kawauchi S, Nakao M, et al: A copy number gain of the $6 \mathrm{p}$ arm is linked with advanced hepatocellular carcinoma. J Pathol 217: 677-684, 2009.

20. Nakao M, Kawauchi S, Furuya T, et al: Identification of chromosomal regions with DNA copy number aberrations associated with node metastasis of colorectal adenocarcinomas based on the array CGH profiles. Cancer Genet Cytogenet 181: 70-76, 2009.

21. Pinto AE, André S, Mendonça E, Silva G and Soares J: Overall survival in advanced breast cancer: relevance of progesterone receptor expression and DNA ploidy in fine-needle aspirates of 392 patients. Int J Biol Markers 18: 7-12, 2003.
22. Spyratos F: DNA content and cell cycle analysis by flow cytometry in clinical samples: application in breast cancer. Biol Cell 78: 69-72, 1993.

23. Michels JJ, Marnay J, Plancoulaine B and Chasle J: Flow cytometry in primary breast carcinomas: prognostic impact of S-phase fraction according to different analysis patterns. Cytometry B Clin Cytom 59: 32-39, 2004.

24. Sourvinos G, Kiaris H, Tsikkinis A, Vassilaros S and Spandidos DA: Microsatellite instability and loss of heterozygosity in primary breast tumours. Tumor Biol 18: 157-166, 1997.

25. Miyakis S and Spandidos DA: Allelic loss in breast cancer. Cancer Detect Prev 26: 426-434, 2002.

26. Cahill DP, Lengauer C, Yu J, et al: Mutations of mitotic checkpoint genes in human cancers. Nature 392: 300-303, 1998.

27. Thompson SL and Compton DA: Examining the link between chromosomal instability and aneuploidy in human cells. J Cell Biol 180: 665-672, 2008.

28. Jallepalli PV and Lengauer C: Chromosome segregation and cancer: cutting through the mystery. Nat Rev Cancer 1: 109-117, 2001.

29. Bannon JH and McGee MM: Understanding the role of aneuploidy in tumorigenesis. Biochem Soc Trans 37: 910-913, 2009.

30. Rao CV, Yamada HY, Yao Y and Dai W: Enhanced genomic instabilities caused by deregulated microtubule dynamics and chromosome segregation: a perspective from genetic studies in mice. Carcinogenesis 30: 1469-1474, 2009.

31. Yuen KW, Warren CD, Chen O, Kwok T, Hieter P and Spencer FA: Systematic genome instability screens in yeast and their potential relevance to cancer. Proc Natl Acad Sci USA 104: 3925-3930, 2007.

32. Kolodner RD, Putnam CD and Myung K: Maintenance of genome stability in Saccharomyces cerevisiae. Science 297: $552-557,2002$ 